\title{
New Applications of Ambient Intelligence
}

\author{
Davide Carneiro and Paulo Novais \\ CCTC/DI - Universidade do Minho \\ Braga, Portugal \\ \{dcarneiro,pjon\}di.uminho.pt
}

\begin{abstract}
Ambient Intelligence emerged more than two decades ago, with the exciting promise of technologically empowered environments that would be everywhere, cater to all our needs, be constantly available, know who we are and what we like, and allow us to make explicit requests using natural means instead of the traditional mouse and keyboard. At a time in which this technological unravelling was expected to have already happened, we still use the mouse and the keyboard. In this paper we make a brief analysis of why is this evolution taking more than initially expected. We then move on to analyse several different projects that are innovative, in the sense that they encompass fields of application that go beyond the initially envisioned, and show the diverse areas that AmI systems may potentially come to change.
\end{abstract}

Keywords: Ambient Intelligence, Future, Innovative Scenarios

\section{Introduction}

In 2001, [5] described four scenarios for Ambient Intelligence, that depicted "what living with 'Ambient Intelligence' might be like for ordinary people in 2010". In the first scenario, 'Maria' - Road Warrior, Maria is someone who is travelling in Europe with a very lightweight hand baggage and no "traditional" personal computing devices: all these have been replaced by the 'P-Com' on her wrist. This device identifies Maria and takes care of going through the airport security, arranges for a rented car, carries her preferences so that the hotel room can adapt to her, among other features. In Scenario 2, 'Dimitrios' and the Digital Me' (D-Me), the main character has a digital avatar embodied in his clothes that is constantly building his profile from the observation of his interactions. D-Me is essentially a very advanced personal assistant, with the autonomy of taking certain decisions in a way that resembles what Dimitrios would do in a similar situation. This way, Dimitrios is free to worry about other issues, while his digital version takes care of certain aspects for him. The third scenario details the daily life of Carmen, in a city-area AmI. Carmen can ask her AmI system to arrange for a ride for work in a shared car, asks her fridge to buy the missing ingredients for a recipe, enjoys an efficient car travelling system that avoids traffic jams and is notified when particular items she is interested in buying are on sale. Essentially, this scenario depicts the advantages of AmI in traffic management, 
sustainability and commerce. Finally, scenario 4 details the hypothetical use of AmI for Social Learning, with an emphasis on scheduling, people's information sharing (e.g. expertise, cv, background) and an ongoing analysis of the mental state of the participants.

Still today, all these scenarios remain in the field of science-fiction. Interestingly enough, the hardware requirements are met (e.g. we can develop a fridge that does online shopping, we can develop an app that shares our background and expertise with people around us). There are however, other questions that hold this development back. Do we really want our fridge to decide on the quality and price of the groceries we buy? Do we really want our personal information to be available to strangers? Indeed, there are many challenges that still remain and go beyond the technological ones. this paper briefly addresses these challenges and then moves on to show that, despite the challenges, the diversity and novelty of AmI applications does not decrease and, still, we expect a near future in which a more seamless integration of technology in our lives takes place.

\section{What is Holding it all Back?}

It is now clear that the development of Ambient Intelligence didn't meet the initially expected pace. This can be attributed to a large (and perhaps increasing) number of challenges. One of these challenges, often disregarded by computer scientists (who form the backbone of AmI development) concerns privacy, identity and security issues. In [14] the authors make a thorough analysis of $70 \mathrm{AmI}$ projects, principally in Europe, concerning these issues. They conclude that in general, current projects present a rather too sunny view of our technological future, ignoring or postponing dealing with some pressing issues. The authors also make an interesting reference to he SWAMI project (Safeguards in a World of Ambient Intelligence) which, against this trend, has constructed what they deemed "dark" scenarios [28], to show how things can go wrong in AmI and where safeguards are needed. Once again, some of these safeguards had already been put forward by [5], while others emerged more recently. As Rouvroy and Brey separately put it, the challenge here is to preserve the individual freedom to build one's own personality without excessive constrains and influences while have control over the aspects of one's identity that one projects on the world $[20,6]$.

Marzano, on a different view, looks at the cultural implications of an unregulated or indiscriminate growth of AmI, making a parallel with the industrial revolution [1]. As later was proved to be, more was not necessarily better at the time: take for instance consequences such as the pollution. Right now, smarter may also not be necessarily better. Indeed, we may simply not want a smart juicer or a talking toaster. The decisions we make now will shape us as a society in the future, as the decisions in the industrial revolution resulted in today's society, for the better and the worse. We must also look at works such as [10], that point out how even the same application of AmI, in the specific case of the paper domestic assistive robots, may be perceived differently, and must thus be 
developed differently, in different cultures, even when these different cultures are within the European context.

Then, and moving to what is most likely the field of the reader, one must also consider the immense technological challenges that are still ahead. One the one hand, we have the challenges that are related to the physical constraints and nature of the necessary hardware. In [12] the authors examine the intricate relationship between the growing need for more computational and communicational power to support increasingly complex services and, at the same time, the need for smaller, more lightweight and efficient devices. It is easy to understand how the objectives of these two fields conflict. Many other technological challenges exist. [8] provide a fairly detailed analysis of these challenges, touching issues such as: (1) the need for increase sensibility, closely related to the assessment of needs and preferences (e.g. a system should be able to know when a new event is important enough to interrupt a user at a given time); (2) the limits of battery life; (3) the simultaneous modelling of several users in the same environment; (4) methods to increase human socialization and interaction in order to avoid the risk of encouraging people to stay home enjoying their AmI systems; (5) the need for increased autonomy in middleware layers; among many others.

Another issue holding back a faster development of AmI that is not so frequently mentioned is the scatter of research efforts. Indeed there are currently many different institutions doing research on very similar topics. When these institutions want to conciliate efforts they may find it difficult to do so since they use different technologies, standards or approaches. Facilitating this integration and interoperability could result in a coming closer of different teams, whom could join efforts and more efficiently work together for the same goal.

\section{Ambient Intelligence: Diversity and Innovation}

Despite the (non-exhaustive) list of challenges presented in the previous section, which seems to only get bigger as research projects approach implementation phases, new fields of application, with extensive lists of advantages, continue to emerge. These prospective advantages are what keeps pushing research forward, despite the numerous challenges. This section presents several fields in which innovative applications of AmI are being developed.

\section{Context-Acquisition}

The gathering, storing, management and provision of contextual information about the user for supporting decision-making are some of the key operations in most AmI systems. Indeed, and depending on the domain and objectives of the application, aspects such as the geographical location, the time of the day, the level of noise or luminosity, the task the user is currently enrolled in or the persons the user is with may be of preponderant importance for the correct provision of services. Particularly, a growing interest exists in the scientific community 
towards the development of approaches that can acquire and mange context information autonomously and with minimum explicit interaction with the user. Examples of such approaches can be found in [18], in which the conflict handling style of users of an Online Dispute Resolution tool is automatically classified, or in [25], in which several examples of context-awareness in mobile tourism guides are presented. More general-purpose approaches can also be found, such as CoWSAMI, a middleware infrastructure that enables context awareness in open ambient intelligence environments, consisting of mobile users and context sources that become dynamically available as the users move from one location to another [3].

\section{Education}

The technological evolution led to significant changes in the Teacher-Student relationship, which does not take part necessarily face-to-face any more and may rely on communication tools that are always poor replacements for our rich communication processes. Interestingly enough, AmI seems to be one possible way of dealing with some of the unaddressed challenges that result from this rapid (and sometimes without control) technological change. One of these challenges is the lack of contextual information of current online communication tools, which are often regarded as cold and impersonal. Teachers and students, that until recently communicated face-to-face, now do it over forums and textbased chats, which makes communication far poorer. In [19], the authors depict a non-invasive way of building contextual information about users of e-Learning tools, that can compile important information for improving the decision-making processes of teachers, namely concerning aspects such as the level of stress or fatigue of students. To overcome similar issues, [17] developed virtual tutoring anthropomorphic characters that provide guidance and motivation to the users and give the feeling that users are being observed, followed and their actions understood. To better accomplish this, the characters are enriched with contextual behavioural models. On another example [2] detail an augmented desk, to be used in the classroom, and improve the learning experience of the student. In a more specific

\section{Robotics}

Robots were always part of the Artificial Intelligence imaginary. Traditionally, each person, in a near future, would presumably have a robotic companion, probably in a humanoid form, taking the role of a multi-faceted personal assistant that would cater to all our needs. Research and technological evolution seem to be pointing to other direction. Indeed, the most likely scenario is the one in which an ecology of agents, generally our traditional appliances and hardware, have communication capabilities and coordinate in order to service us, through some kind of enabling middleware [13]. Robots, literally, can also be conceived that behave similarly, sharing our environment and moving naturally through it while providing their services [23]. A mixed scenario is also envisioned by 
researchers in which traditional appliances and robots, in the true sense of the word, coordinate their efforts in order to accomplish their goals and, in doing so, implement AmI [22].

\section{Health}

The Healthcare sector is currently one of the technologically most advanced ones. It thus comes as no surprise that many different projects can be found in this field. Two main trends can be identified: the provision of healthcare services at home and the support of healthcare in the hospital. In both scenarios, the main aim is to decrease healthcare costs while making it more accessible, personalized and available [21]. In the home scenario, most of the projects target the support of the elderly, the so-called assisted living, to provide them with more independence and quality of life [7]. In the healthcare institution, one can find projects to deal with very specific types of scenarios (e.g. Alzheimer patients [11]) as well as more generic ones such as the use of discrete hidden Markov models for classifying the activities of healthcare practitioners [24]. Concerning the very particular case of mental disabilities, [26] presents a tool for the assessment of cognitive skills of mentally disabled working people, which can be quite important for improving disabled people's performance and self-esteem in their workplaces.

\section{Transportation}

Given that we spend a significant part of our lives travelling back and forth, transportation is another field in which the use of Ambient Intelligence could translate into multiple advantages, as pointed out by [8]: (1) train stations, buses, and cars can be equipped with technology that can provide fundamental knowledge about how the system is performing; (2) identification of potential improvements by using the system more effectively, that can result in the improvement of the experience of people; (3) GPS-based spatial location of public transportation vehicles and services; (4) vehicle identification and image processing to make transport more fluent and hence more efficient and safe, or to detect situations of interest in busy conditions [27], among many others. The coming together of these and other technological evolutions could result in significant improvements in currently challenged transportation networks.

\section{Emergency Services}

Emergency services and their organization constitute a complex and critical field in which one can also find applications for Ambient Intelligence. Here, the main objectives are the provision of mission-critical knowledge in real-time and the coordination of the different actors, as addressed by the AMIRA project [4]. [16] further discuss the possibilities of AmI in emergency services, namely their potential role in the rapid organization of virtual response teams as well as the support for the communication and coordination of the members of such teams, even in the cases in which they span different countries. 


\section{Culture}

Ambient Intelligence is also being researched as a way of implementing better access to sources of culture. An example of a project in a domestic environment is GENIO, that besides other services provides the user with easy and intuitive access to music, videos and pictures, accessible through voice commands "heard" by the system from a microphone worn by the user on the chest [15]. In an outdoors example one can find DALICA: a project that provided access to information about geographically spread monuments in a geographical area in Villa Adriana, Italy [9]. The users can visit the different stone monuments and automatically receive information on the smartphone as they approach them. In the overall, AmI can make access to culture more intuitive, personalized and automatized, making the whole experience more pleasant.

\section{Conclusions}

Ambient Intelligence has not grown as fast as initially expected. The natural interfaces, always present and proactive assistants or the seamless experience still seem far from reality. Several challenges have been pointed out that can explain, at least in part, this delay. These include technological (mostly at the level of integration), cultural, and safety and privacy-related challenges.

Interestingly enough, this does not prevent new ideas and new fields of application from emerging. Indeed, we continue to see an increasing diversity of the areas in which AmI would result in amazing advantages. These areas include, as depicted in this paper, sensitive ones such as healthcare, transportation or emergency services as well as more "traditional" ones such as education or home assistants.

It seems rather contradictory that a field with so many challenges still to be addressed continues to expand, to grow in different directions, most likely finding additional new challenges. The truth is that this expansion cannot stop. The real possibilities of AmI and the advantages of its application in so many fields are what continues to push research forward. There is the need to show what can actually be done once the challenges are overcome. Once these challenges are overcome and the technology finally unravels as expected, what was once science fiction will become reality, giving the feeling of a new technological evolution.

\section{Acknowledgements}

This work is part-funded by ERDF - European Regional Development Fund through the COMPETE Programme (operational programme for competitiveness) and by National Funds through the FCT - Fundação para a Ciência e a Tecnologia (Portuguese Foundation for Science and Technology) within project FCOMP-01-0124-FEDER-028980 (PTDC/EEI-SII/1386/2012). This work is partfunded by National Funds through the FCT - Fundação para a Ciência e a Tecnologia (Portuguese Foundation for Science and Technology) within projects PEst-OE/EEI/UI0752/2011. 


\section{References}

1. Aarts, E.H., Marzano, S. (Eds.). The new everyday: Views on ambient intelligence. 010 Publishers. (2003)

2. Antona, M., Margetis, G., Ntoa, S., Leonidis, A., Korozi, M., Paparoulis, G., Stephanidis, C.: Ambient Intelligence in the classroom: an augmented school desk. In Proceedings of the 2010 AHFE International Conference, pp. 17-20 (2010)

3. Athanasopoulos, D., Zarras, A. V., Issarny, V., Pitoura, E., Vassiliadis, P. CoWSAMI: Interface-aware context gathering in ambient intelligence environments. Pervasive and Mobile Computing, 4(3), pp. 360-389. (2008).

4. Bergmann, R.: Ambient intelligence for decision making in fire service organizations. In Ambient Intelligence, pp. 73-90. Springer Berlin Heidelberg (2007)

5. Bogdanowicz, M., Scapolo, F., Leijten, J., Burgelman, J. C.: Scenarios for ambient intelligence in 2010, pp. 3-8. Office for official publications of the European Communities (2001)

6. Brey, P. Freedom and privacy in ambient intelligence. Ethics and Information Technology, 7(3), pp. 157-166 (2005)

7. Cesta, A., Cortellessa, G., Pecora, F., Rasconi, R. Exploiting scheduling techniques to monitor the execution of domestic activities. Intelligenza Artificiale, 2, 4 (2005)

8. Cook, D. J., Augusto, J. C., Jakkula, V. R. Ambient intelligence: Technologies, applications, and opportunities. Pervasive and Mobile Computing, 5(4), 277-298. (2009)

9. Costantini, S., Mostarda, L., Tocchio, A., Tsintza, P.: DALICA: Agent-based ambient intelligence for cultural-heritage scenarios. Intelligent Systems, IEEE, 23(2), pp. 34-41 (2008)

10. Cortellessa, G., Scopelliti, M., Tiberio, L., Svedberg, G. K., Loutfi, A., Pecora, F. A cross-cultural evaluation of domestic assistive robots. In Proceedings of the AAAI Fall Symposium on AI and Eldercare. (2008)

11. Corchado, J. M., Bajo, J., Abraham, A.: GerAmi: Improving healthcare delivery in geriatric residences. Intelligent Systems, IEEE, 23(2), pp. 19-25 (2008)

12. De Man, H.: Ambient intelligence: gigascale dreams and nanoscale realities. In Solid-State Circuits Conference. Digest of Technical Papers. ISSCC. IEEE International, pp. 29-35, IEEE. (2005)

13. Encarnaçao, J. L., Kirste, T.: Ambient intelligence: Towards smart appliance ensembles. In From Integrated Publication and Information Systems to Information and Knowledge Environments (pp. 261-270). Springer Berlin Heidelberg (2005)

14. Friedewald, M., Vildjiounaite, E., Punie, Y., Wright., D.: The Brave New World of Ambient Intelligence: An Analysis of Scenarios regarding Privacy, Identity and Security Issues. Security in Pervasive Computing. Proceedings of the third International Conference, SPC 2006, York, UK, April 18-21, 2006. Ed. Clark, J. A. et al. Heidelberg, Berlin: Springer, pp. 119-133 (2006)

15. Gárate, A., Herrasti, N., López, A.: GENIO: an ambient intelligence application in home automation and entertainment environment. In Proceedings of the 2005 joint conference on Smart objects and ambient intelligence: innovative contextaware services: usages and technologies (pp. 241-245). ACM (2005)

16. Jones, V., Karagiannis, G., Heemstra de Groot, S.: Ad hoc networking and ambient intelligence to support future disaster response (2005)

17. Ndiaye, A., Gebhard, P., Kipp, M., Klesen, M., Schneider, M., Wahlster, W. Ambient intelligence in edutainment: Tangible interaction with life-like exhibit guides. In Intelligent technologies for interactive entertainment (pp. 104-113). Springer Berlin Heidelberg. (2005) 
18. Novais P., Carneiro D., Gomes M., Neves J.: Behavioural and Context Analysis in an Online Dispute Resolution Environment. In: Proceedings of the Sixth International Workshop on Juris-Informatics (JURISIN 2012), hosted by the Fourth JSAI International Symposia on AI (2012)

19. Rodrigues, M., Gonçalves, S., Carneiro, D., Novais, P., Fdez-Riverola F.: Keystrokes and Clicks: Measuring Stress on E-learning Students. Management Intelligent Systems - Advances in Intelligent Systems and Computing Volume 220, pp 119-126 (2013)

20. Rouvroy, A.: Privacy, data protection, and the unprecedented challenges of ambient intelligence. Studies in ethics, law, and technology, 2(1) (2008)

21. Sadri, F.: Ambient intelligence: A survey. ACM Computing Surveys (CSUR), 43(4), $36(2011)$

22. Saffiotti, A., Broxvall, M.: PEIS ecologies: Ambient intelligence meets autonomous robotics. In Proceedings of the 2005 joint conference on Smart objects and ambient intelligence: innovative context-aware services: usages and technologies (pp. 277281). ACM (2005)

23. Sanfeliu, A., Hagita, N., Saffiotti, A.: Network robot systems. Robotics and Autonomous Systems, 56(10), 793-797 (2008)

24. Sánchez, D., Tentori, M., Favela, J.: Activity recognition for the smart hospital. Intelligent Systems, IEEE, 23(2), pp. 50-57 (2008)

25. Schwinger, W., Grün, C., Pröll, B., Retschitzegger, W., Schauerhuber, A.: Contextawareness in mobile tourism guides - A comprehensive survey. Rapport Technique. Johannes Kepler University Linz (2005)

26. Vilaro, A., Orero, P.: User-centric cognitive assessment. Evaluation of attention in special working centres: from paper to Kinect. Advances in Distributed Computing and Artificial Intelligence Journal. Vol 1, No 7: Special Issue 7 (2013)

27. Velastin, S.A., Boghossian, B.A., Lo, B. P., Sun, J., Vicencio-Silva, M. A.: PRISMATICA: toward ambient intelligence in public transport environments. Systems, Man and Cybernetics, Part A: Systems and Humans, IEEE Transactions on, 35(1), pp. 164-182 (2005)

28. Wright, D.: The dark side of ambient intelligence. info, 7(6), pp. 33-51 (2005) 